\title{
QUEIXA DE PROBLEMAS COM A MEMÓRIA E $O$ DIAGNÓSTICO DE DEMÊNCIA
}

\author{
OSVALDO P. ALMEIDA*
}

\begin{abstract}
RESUMO - O processo de envelhecimento é acompanhado de declínio em algumas habilidades intelectuais, embora apenas em alguns casos essa deterioração prossiga no sentido de evolução para demência. Este estudo teve como objetivo investigar a associação entre a queixa subjetiva de dificuldades com a memória e o diagnóstico de demência entre os idosos atendidos em um ambulatório de saúde mental. Todos os casos novos com mais de 60 anos de idade avaliados na Unidade de Idosos do Ambulatório de Saúde Mental da Santa Casa de São Paulo entre fevereiro e dezembro de 1997 foram incluídos no estudo. Eles foram avaliados de forma sistemática com o SRQ-20, MMSE e avaliação clínica para realização do diagnóstico de acordo com a CID-10. Dos 220 idosos avaliados, 59,1\% queixavam-se de problemas com a memória. Setenta e um por cento dos que se queixavam eram do sexo feminino, embora não houvesse associação clara entre sexo, educação, estado civil e viver só com dificuldades subjetivas de memória. Idosos com esse tipo de queixa tendiam a apresentar escores maiores no SRQ-20 ( $\mathrm{p}=0,122)$. A sensibilidade da queixa de memória para o diagnóstico de demência foi 76,2\%, a especificidade foi $47,8 \%$, o valor preditivo positivo foi $36,9 \%$ e o valor preditivo negativo foi $83,3 \%$. Conclui-se que queixas de problemas com a memória são frequentes entre os idosos, e que sintomas ansiosos e depressivos podem ser um pouco mais freqüentes nesses pacientes. A vivência subjetiva de perda de memória possui baixo valor preditivo para o diagnóstico de demência. A identificação de populações de risco para o desenvolvimento de demência deve basear-se em outros métodos de investigação como neuroimagem e genética.
\end{abstract}

PALAVRAS-CHAVE: demência, depressão, ansiedade, memória, queixa de memória, envelhecimento, idoso, declínio cognitivo associado ao envelhecimento, declínio da memória associado ao envelhecimento.

\section{Memory complaints and the diagnosis of dementia}

ABSTRACT - Ageing is often associated with the decline of some cognitive abilities, although in most cases these losses do not progress to full blown dementia. The current study aimed to evaluate the association between subjective memory complaint and the diagnosis of dementia among the elderly assessed at the Old Age Unit of the Department of Mental Health of Santa Casa de São Paulo-Brazil between February and December 1997. Subjects were assessed with the SRQ-20 and MMSE. Further clinical information was also collected to allow for the diagnosis of mental disorders according to ICD-10 diagnostic guidelines. Fifty-nine percent of the 220 patients included in the study complained of memory difficulties. Seventy-one percent of the complainers were women, although there was no clear statistical association between sex, education, marital status and living conditions with the memory complaint. There was a trend for memory complainers to present with higher scores on the SRQ-20 ( $\mathrm{p}=0.122)$. The complaint of memory difficulties had a sensitivity of $76.2 \%$, specificity of $47.8 \%$, positive predictive value of $36.9 \%$, and negative predictive value of $83.3 \%$ for the diagnosis of dementia. Memory complaints are frequent among the elderly, particularly among those with more severe depressive and anxiety symptoms. The subjective experience of memory difficulties has a low predictive value for the diagnosis of dementia. The identification of "at risk" cases should, instead, be based on new neuroimaging and genetic methods.

KEY WORDS: dementia, depression, anxiety, memory, memory complaint, ageing, elderly, age associated cognitive decline, age associated memory impairment.

*Unidade de Idosos do Departamento de Saúde Mental da Santa Casa de São Paulo; bolsista nível IIa do CNPq. Aceite: 5-março-1998.

Dr. Osvaldo P. Almeida - Unidade de Idosos do Departamento de Saúde Mental da Santa Casa de São Paulo Rua Dr. Cesário Motta Jr. 112 - 01277-000 São Paulo SP. FAX 011220 2008; E-mail: osvalm@ibm.net 
O desenvolvimento de dificuldades progressivas com a memória durante o processo de envelhecimento tem sido interpretado de diferentes formas ao longo dos anos. Alguns autores consideram que o declínio intelectual não faz parte do processo de envelhecimento normal, enquanto outros sugerem que "a mente se forma e amadurece junto com o corpo; cresce com ele e envelhece com ele... quando o corpo sucumbe sob a poderosa força do tempo, o intelecto tropeça, a língua balbucia, a inteligência perde o ritmo; tudo é querer e fracassar ao mesmo tempo" (Lucrecius, citado por Berrios ${ }^{1}$ ). O próprio Alois Alzheimer, ao descrever a doença que hoje recebe seu nome, sugeriu que o quadro demencial por ele observado representava uma "forma de envelhecimento acelerado" 2 . De fato, há várias evidências de que o desempenho intelectual do idoso apresenta discreta deterioração em tarefas que exigem maior velocidade e flexibilidade no processamento de informações ${ }^{3}$. Com o passar do tempo a memória também pode sofrer algum comprometimento em relação a fases anteriores da vida, particularmente a memória processual e a lembrança livre ${ }^{3}$. Será, portanto, que a vivência subjetiva de declínio da memória indica sempre a presença de um quadro demencial em evolução?

Acredita-se hoje que a deterioração intelectual característica da doença de Alzheimer (DA) não é uma consequência inevitável do processo de envelhecimento. Fatores genéticos (mutações nos genes da "proteína precursora de amilóide", "presenilina 1" e "presenilina 2") parecem desempenhar papel fundamental nas formas familiares da doença com início pré-senil, podendo também funcionar como fatores de risco em alguns casos de início tardio (apolipoproteína E, butirilcolinesterase-K, mutações no cromossomo 12$)^{4-6}$. Em outras palavras, fatores genéticos e ambientais específicos ${ }^{7}$ contribuem para que alguns idosos (mas não todos) tornem-se particularmente vulneráveis ao desenvolvimento de declínio intelectual.

Passou-se a buscar, então, critérios capazes de discriminar queixas de memória associadas ao envelhecimento normal daquelas indicativas de processo demencial. Diagnósticos como "esquecimento benigno da senescência"8, "comprometimento da memória associado ao envelhecimento", ou "declínio cognitivo associado à idade" 10 foram introduzidos com esse objetivo" . Estudos epidemiológicos indicam que 4-54\% dos idosos apresentam queixas relacionadas à memória ${ }^{12-}$ ${ }^{16}$. Entre aqueles que se queixam e não preenchem critérios para qualquer diagnóstico neuropsiquiátrico encontram-se mais frequentemente as mulheres com elevado nível de escolaridade que moram só e que relatam história de demência na família ${ }^{17}$. Além disso, a presença de sintomas depressivos e ansiosos parece ser outra importante fonte de vivência subjetiva de deterioração da memória não devido a demência ${ }^{18}$.

Este estudo teve como objetivo verificar as associações clínicas das queixas de problemas com a memória em uma população de pacientes idosos atendidos em um ambulatório de saúde mental em São Paulo, Brasil. Para tal, buscou-se testar as seguintes hipóteses: (1) queixas de problemas com a memória são mais frequentes entre as mulheres; (2) sintomas depressivos e ansiosos são mais frequentes entre os pacientes que se queixam de dificuldades com a memória; (3) queixas relacionadas à memória apresentam baixo valor preditivo para o diagnóstico de demência.

\section{MÉTODOS}

Todos os casos novos atendidos no ambulatório da Unidade de Idosos (UNID) do Departamento de Saúde Mental da Santa Casa de São Paulo entre 25 de fevereiro e 9 de dezembro de 1997 foram incluídos no estudo. A UNID atende pacientes com idade igual ou superior a 60 anos que procuram o serviço de forma espontânea ou através de encaminhamento de outros profissionais de saúde. A estruturação das consultas médicas desses pacientes foi descrita de forma detalhada em outra publicação ${ }^{19}$. Resumidamente, todos os pacientes são entrevistados com o SRQ-20 (Self Reporting Questionnaire) ${ }^{20,21}$ e o Mini-Exame do Estado Mental - MMSE22 para a investigação do estado mental atual. Uma questão suplementar foi adicionada à entrevista para verificar a vivência subjetiva de dificuldades com a memória: "Você tem tido problemas com sua memória?", sendo as respostas possíveis "sim" ou "não". Além disso, uma entrevista aberta é utilizada para a coleta de informações clínicas suplementares que possibilitem a realização de um diagnóstico sindrômico de acordo com as diretrizes da CID-1023. 
Os dados foram analisados com o pacote estatístico "SPSS versão 6.0 para Windows". Análise de tabelas de contingência foi utilizada para a investigação de variáveis categoriais, com o resultado estatístico sendo distribuído como qui-quadrado de Pearson $\left(\chi^{2}\right)$. Teste $t$ de Student foi utilizado para a comparação das médias entre dois grupos. O odds ratio foi calculado para estimar o risco relativo associado a algumas variáveis (por exemplo: sexo). Intervalos de confiança de $95 \%$ foram estimados para as médias (CI), diferença entre as médias $\left(\mathrm{CI}_{\mathrm{d}}\right)$ e para o odds ratio $\left(\mathrm{CI}_{\mathrm{OR}}\right)$. Taxas de sensibilidade, especificidade, valor preditivo positivo e valor preditivo negativo foram estimadas a partir de tabelas de contingência $2 \mathrm{X} 2$.

\section{RESULTADOS}

Duzentos e cinquenta e nove casos novos foram atendidos no ambulatório da UNID durante o período de coleta de informações para o estudo. Trinta e um $(12,0 \%)$ desses pacientes foram excluídos da análise por não ter sido possível estabelecer o diagnóstico sindrômico desses casos na primeira consulta. Outros 8 pacientes $(3,1 \%)$ foram excluídos do estudo por não terem respondido à pergunta referente aos problemas de memória ou porque essa resposta não foi adequadamente registrada. Os resultados aqui apresentados referem-se, portanto, a um total de 220 idosos avaliados nos ambulatórios da UNID. Estes tinham idade média de 69,48 anos (CI=68,44 a 70,53), sendo $69,6 \%$ deles do sexo feminino.

Cento e trinta $(59,1 \%)$ dos pacientes avaliados queixaram-se de problemas com a memória. A Tabela 1 resume as associações clínicas entre queixas relacionadas à memória e dados demográficos básicos. Não se observou diferença significativa entre pacientes sem e com queixa de problemas de memória no Mini-Exame do Estado Mental (MMSE=23,30 e 22,07 respectivamente; $\mathrm{t}=1,25, \mathrm{p}=0$,212; $\mathrm{CI}_{\mathrm{d}}=-0,71 \mathrm{a} 3,18$ ), embora fosse possível observar tendência para escores mais elevados no SRQ-20 entre os pacientes queixosos (escores $=8,11$ e 9,10 respectivamente; $t=-1,55, p=0,122 ; \mathrm{CI}_{d}=-2,27 \mathrm{a}$ $0,27)$. Sessenta e três $(28,6 \%)$ pacientes preenchiam os critérios para demência de acordo com a CID-10, enquanto $81(36,8 \%)$ satisfaziam os critérios para o diagnóstico de episódio depressivo maior ou distimia. Pacientes com diagnóstico de demência queixavam-se significativamente mais

Tabela 1. Dados demográficos básicos de idosos com e sem queixas relacionadas à memória.

\begin{tabular}{|c|c|c|c|c|}
\hline & $\begin{array}{l}\text { Sem queixa } \\
\qquad(n=90)\end{array}$ & $\begin{array}{l}\text { Com queixa } \\
\qquad(\mathrm{n}=130)\end{array}$ & $\chi^{2}, \mathrm{t}, \mathrm{z}$ & $\mathrm{p}$ \\
\hline Idade (média) & 68,88 & 69,90 & 0,95 & $\begin{array}{c}0,344 \\
\mathrm{CI}_{\mathrm{d}}=-3,15 \text { a } 1,10\end{array}$ \\
\hline Sexo feminino $(\%)$ & 66,7 & 71,5 & 0,60 & $\begin{array}{c}0,440 \\
\mathrm{CI}_{\mathrm{OR}}=0,45 \text { a } 1,42\end{array}$ \\
\hline $\begin{array}{l}\text { Estado civil casado ou } \\
\text { vivendo maritalmente }(\%)\end{array}$ & $\begin{array}{c}55,7 \\
(\mathrm{dp}=2)\end{array}$ & $\begin{array}{c}49,6 \\
(\mathrm{dp}=1)\end{array}$ & 0,77 & $\begin{array}{c}0,380 \\
\mathrm{CI}_{\mathrm{OR}}=0,46 \text { a } 1,35\end{array}$ \\
\hline $\begin{array}{l}\text { Escolaridade }(\%) \\
\text { nenhuma } \\
\text { primário } \\
\text { ginásio } \\
\text { colégio } \\
\text { superior }\end{array}$ & $\begin{array}{c}23,3 \\
54,4 \\
6,7 \\
11,1 \\
4,4\end{array}$ & $\begin{array}{c}23,3 \\
45,0 \\
13,9 \\
11,6 \\
6,2 \\
(\mathrm{dp}=1)\end{array}$ & 3,86 & 0,426 \\
\hline $\begin{array}{l}\text { Número de pessoas } \\
\text { morando só }(\%)\end{array}$ & $\begin{array}{c}14,8 \\
(\mathrm{dp}=2)\end{array}$ & $\begin{array}{c}20,9 \\
(\mathrm{dp}=1)\end{array}$ & 1,32 & $\begin{array}{c}0,251 \\
\mathrm{CI}_{\mathrm{OR}}=0,75 \text { a } 3,12\end{array}$ \\
\hline
\end{tabular}

$\mathrm{CI}_{\mathrm{d}}$, intervalo de confiança de $95 \%$ da média; $\mathrm{CI}_{\mathrm{OR}}$, intervalo de confiança de $95 \%$ do "odds ratio"; dp, número de casos perdidos para análise. 
de problemas com a memória do que pacientes deprimidos (76,3\% e 51,8\% respectivamente; $\chi^{2}=8,96$, $\mathrm{df}=1, \mathrm{p}=0,003 ; \mathrm{OR}=2,97, \mathrm{CI}_{\mathrm{OR}}=1,45$ a 6,09$)$. Não se observou diferença nos escores do MMSE entre os pacientes demenciados sem e com queixa de problemas com a memória (MMSE=16,00 e 16,55 respectivamente; $\left.\mathrm{t}=-0,249, \mathrm{p}=0,804 ; \mathrm{CI}_{\mathrm{d}}=-4,95 \mathrm{a} 3,85\right)$. A sensibilidade da queixa de memória para o diagnóstico de demência foi $76,2 \%$, a especificidade foi $47,8 \%$, o valor preditivo positivo foi $36,9 \%$ e o valor preditivo negativo foi $83,3 \%$.

\section{DISCUSSÃO}

A queixa de dificuldades com a memória é frequente entre idosos, tendo sido observada em $59,1 \%$ dos 220 casos novos atendidos nos ambulatórios da UNID. Estudos realizados em outros países indicaram que esse tipo de queixa é mais frequente entre as mulheres ${ }^{17}$. Ao longo dos últimos anos várias hipóteses foram testadas com o objetivo de explicar esse padrão de comportamento - a mais consistente entre elas é a que se refere à privação de estrógeno no climatério. Mulheres no período pós-menopausa podem apresentar discreto declínio no desempenho em testes de memória episódica, o qual pode ser revertido através de terapia de reposição estrogênica ${ }^{24}$. Esses achados sugeriam, portanto, que o estrógeno deve ter uma ação sobre o sistema nervoso central (SNC) que vai além de sua função sexual. De fato, o estrógeno induz aumento: na atividade da acetil-colinotransferase; no número de receptores muscarínicos, de axônios e dendritos, e de receptores NMDS no hipocampo; da expressão do NGF (fator neurotrófico de crescimento); e do funcionamento de sistemas serotonérgicos e noradrenérgicos ${ }^{25,26}$. Várias dessas ações do estrógeno têm, possivelmente, impacto sobre o funcionamento cognitivo das mulheres no período após a menopausa e explicam, ao menos em parte, a maior frequência de queixas relacionadas à memória no sexo feminino. Além disso, há evidências consistentes de que a privação estrogênica pode tornar as mulheres mais vulneráveis ao desenvolvimento da DA. Tang e colaboradores ${ }^{27}$ acompanharam por 5 anos uma amostra de 1124 mulheres idosas vivendo na comunidade. Eles observaram que aquelas submetidas a terapia de reposição estrogênica (13,8\% do total) apresentavam declínio de 2,5 vezes no risco relativo de desenvolverem DA.

Os resultados deste estudo indicam que há predominância de mulheres entre aqueles que se queixam de dificuldades com a memória (71,5\%), embora não fosse possível demonstrar diferença significativa na distribuição por sexo entre aqueles com e sem queixa. Essa aparente contradição pode ser parcialmente explicada pelo fato de mais de $2 / 3$ dos pacientes avaliados serem do sexo feminino. Em outras palavras, a absoluta maioria de mulheres na população estudada pode ter dificultado a detecção de diferenças relacionadas ao sexo. Além disso, vale lembrar que essa preponderância de mulheres entre pacientes idosos não parece ser específica de serviços de saúde mental. Um estudo recente de Almeida e colaboradores ${ }^{28}$ revelou que $83,5 \%$ dos pacientes com mais de 60 anos de idade atendidos em um centro de saúde (atenção primária) são do sexo feminino, embora o motivo que leva as mulheres a procurarem os serviços de saúde com maior frequência ainda seja objeto de especulação ${ }^{28}$.

Outras variáveis como viver só, não ser casado, e apresentar nível educacional elevado também têm sido associadas a maior frequência de dificuldade subjetiva com a memória ${ }^{17,29}$, embora o presente estudo fosse incapaz de demonstrar esse perfil entre os pacientes avaliados. Estudos realizados no Brasil sugerem que os idosos do país vivem, na maior parte das vezes, com algum familiar ${ }^{30}$. Este é um dado que contrasta com os observados em países do hemisfério norte, onde uma parcela significativa dessa população vive sozinha ${ }^{31}$. Assim, o tipo de estrutura social na qual o idoso brasileiro está inserido é diferente daquele observado em países desenvolvidos, o que poderia explicar as diferentes associações demográficas observadas na presente amostra. É possível, ainda, que os idosos atendidos em nível ambulatorial no Brasil não representem adequadamente o total da população de idosos vivendo na comunidade - a presença de um familiar próximo ao paciente pode facilitar o encaminhamento do indivíduo para atendimento médico e, como consequência, apenas aqueles idosos com maior suporte social são atendidos nesse tipo de serviço. 
Outra peculiaridade do serviço de saúde pública do Brasil é o fato de este ser utilizado principalmente pela parcela mais desprovida da população ${ }^{19}$. Portanto, não chega a ser uma surpresa que o nível educacional desses pacientes seja baixo. De fato, mais de $2 / 3$ de nossa amostra era constituída de indivíduos que receberam no máximo educação elementar. Essa é, possivelmente, a explicação para o fato de não termos observado nenhum tipo de associação entre queixa de dificuldades com a memória e nível educacional — não havia número suficiente de idosos com nível superior de escolaridade em nossa amostra.

A presença de sintomas depressivos e ansiosos tem sido frequentemente associada a queixas relacionadas à memória ${ }^{12,13,32,33}$. De fato, mais de metade dos pacientes atendidos na UNID com episódio depressivo maior ou distimia queixavam-se de dificuldades com a memória. Além disso, pacientes queixosos tendiam a exibir escores mais elevados no SRQ-20 (o qual mede predominantemente sintomas ansiosos e depressivos), sugerindo que a presença dessas queixas é muitas vezes indicativa da presença de depressão ou ansiedade. Apesar de a queixa de memória ser pouco específica $(47,8 \%)$ e ter baixo valor preditivo para o diagnóstico de demência $(36,9 \%)$, é preciso cuidado para não a desvalorizar - 76,3\% daqueles com diagnóstico de demência referem dificuldades nessa área (valor preditivo negativo $=83,3 \%$ ). Ou seja, muitas vezes a queixa de problemas com a memória é indicativa de processo demencial.

É importante destacar que a metodologia utilizada neste estudo para avaliar a presença de dificuldades com a memória é diferente daquela que serve de base ao diagnóstico de "comprometimento da memória associado ao envelhecimento" (AAMI). O diagnóstico de AAMI tem como base a queixa espontânea de dificuldades com a memória por parte do paciente ${ }^{34}$, enquanto nossa abordagem do problema incluía o questionamento ativo de dificuldades nessa área. É possível que pacientes demenciados não se queixem espontaneamente sobre problemas de memória, mas que o número daqueles que confirmam a presença de dificuldades nessa área aumente sensivelmente quando os eles são inquiridos de forma direta. É interessante notar, ainda, que a gravidade do quadro demencial (conforme medido pelo MMSE) não teve associação íntima com a percepção subjetiva de dificuldades com a memória. De fato, Green e colaboradores ${ }^{35}$ observaram que percepção dos próprios déficits em pacientes com DA é altamente variável, sugerindo que essa capacidade crítica não depende apenas da gravidade do quadro demencial.

A ausência de padrões clínicos confiáveis capazes de revelar os pacientes que eventualmente evoluirão para um quadro demencial foi confirmada por estudos que mostraram que mais de $90 \%$ daqueles que se queixavam continuavam bem após vários meses ou anos de seguimento ${ }^{36-38}$. Isso fez com que vários pesquisadores buscassem medidas que tivessem maior valor preditivo de evolução para demência que a simples queixa de memória. Spanó e colaboradores ${ }^{39}$ observaram que os pacientes que se queixavam de dificuldades com a memória apresentavam um volume intermediário entre controles e pacientes com DA da fissura de Sylvius em medidas de tomografia computadorizada de crânio. A utilização de ressonância nuclear magnética revelou que indivíduos com diagnóstico de AAMI apresentam redução de $14 \%$ no volume do hipocampo quando comparados a controles normais ${ }^{40}$. Além disso, estudos utilizando métodos de neuroimagem funcional (e.g. SPET) indicam que indivíduos classificados como AAMI apresentam valores intermediários de fluxo sanguíneo cerebral em região têmporo-parietal quando comparados a controles e portadores da $\mathrm{DA}^{41}$. Mais recentemente, Reiman e colaboradores ${ }^{42}$ demonstraram que adultos normais portadores do alelo $\varepsilon 4$ da apolipoproteína E apresentam padrão de redução do metabolismo cortical que tem características semelhantes àquele observado na DA. Se esses resultados forem confirmados passaremos a ter medidas mais confiáveis do que a simples queixa de dificuldades com a memória para identificar populações com grande risco de evolução para demência.

\section{CONCLUSÃO}

Queixas de dificuldades com a memória são frequentes entre os idosos atendidos em um ambulatório de saúde mental, embora essas possuam baixa especificidade e valor preditivo para o 
diagnóstico de demência. Apesar disso, a confirmação por parte do paciente desse tipo de dificuldade não deve ser desconsiderada, já que mais de 3/4 dos portadores de demência relatam problemas com a memória. Além disso, pacientes com esse tipo de queixa tendem a apresentar mais sintomas ansiosos e depressivos, sugerindo que esses sintomas devem ser sempre investigados (e tratados) quando o paciente relata que sua memória está ruim.

O uso de uma única pergunta ("Você tem tido problemas com sua memória?") para investigar possiveis dificuldades com a memória é, certamente, um screening insatisfatório para a detecção de quadros demenciais. A utilização dos novos métodos de neuroimagem e genética pode mostrar-se mais útil na identificação de indivíduos com alto risco de evolução para demência.

Agradecimentos - O autor agradece a valiosa contribuição do Dr. Sérgio Tamai e Dra. Regiane Garrido na coleta dos dados e de Leonete Faria Arruda na organização do banco de dados.

\section{REFERÊNCIAS}

1. Berrios GE. Dementia and aging since the nineteenth century. In Huppert FA, Brayne C, O'Connor DW (eds). Dementia and normal aging. Cambridge: Cambridge Univ Press, 1994:15-40.

2. Alzheimer A. "Über eine eigenartige Erkrankung der Hirnrinde" 1907. Translated by Jarvik L, Greenson H, About a peculiar disease of the cerebral cortex. Alzh Dis Assoc Disord 1987;1:7-8.

3. Morris RG. Cognition and ageing. In Jacoby R, Oppenheimer C (eds). Psychiatry in the elderly. Oxford: Oxford Univ Press, 1997:37-62.

4. Almeida OP. Biologia molecular da doença de Alzheimer: uma luz no fim do túnel? Rev Assoc Med Bras 1997;43:77-81.

5. Almeida OP, Shimokomaki CM. ApoE4 and Alzheimer's disease in São Paulo - Brazil. Arq Neuropsiquiatr 1997;55:1-7.

6. Lehmann DJ, Johnston C, Smith AD. Synergy between the genes for butyrylcholinesterase K variant and apolipoprotein E4 in late-onset confirmed Alzheimer's disease. Hum Mol Genet 1997;6:1933-1936.

7. Almeida OP, Bottino C. Em busca das causas da doença de Alzheimer. Rev ABP-APAL 1996;18:117-124.

8. Kral VA. Senescent forgetfulness: benign and malignant. Canad Med Ass J 1962;86:257-260.

9. Crook TH, Feher EP, Larrabee GJ. Assessment of memory complaint in age-associated memory impairment: the MAC-Q. Int Psychogeriat 1992;4:165-176.

10. American Psychiatric Association. Diagnostic criteria from DSM-IV. Washington: American Psychiatric Association, 1994.

11. Levy R. Declínio cognitivo associado ao envelhecimento. In Almeida OP, Nitrini R (eds). Demência. São Paulo: Fundo Editorial Byk, 1995:101-105.

12. Barker A, Jones R, Jennison C. A prevalence study of age-associated memory impairment. Br J Psychiatry 1995;167:642648.

13. Christensen H, Henderson AS, Jorm AF, et al. ICD-10 mild cognitive disorder: epidemiological evidence on its validity. Psychol Med 1995;25:105-120.

14. Coria F, Gomez de Caso JA, Minguez L, et al. Prevalence of age-associated memory impairment and dementia in a rural community. J Neurol Neurosurg Psychiatry 1993;53:973-976.

15. Ebly EM, Hogan DB, Parhad IM. Cognitive impairment in the nondemented elderly: results from the Canadian study of health and aging. Arch Neurol 1995;52:612-619.

16. Koivisto K, Reinikainen KJ, Hanninen T, et al. Prevalence of age-associated memory impairment in a randomly selected population from eastern Finland. Neurology 1995;45:741-747.

17. Almeida OP, Hill K, Howard R, et al. Demographic and clinical features of patients attending a memory clinic. Int J Geriat Psychiatry 1993;8:497-501.

18. Grut M, Jorm AF, Fratiglioni L, et al. Memory complaints of elderly people in a population survey: variation according to dementia stage and depression. J Am Geriat Soc 1993;41:1295-1300.

19. Almeida OP, Garrido R, Tamai S. Unidade para idosos (UNID) do Departamento de Saúde Mental da Santa Casa de São Paulo: características clínicas de pacientes atendidos em nível ambulatorial. J Bras Psiquiat (in press).

20. Harding TW, Climent C, Diop M, et al. The WHO collaborative study on strategies for extending mental health care II: the development of new research methods. Am J Psychiatry 1983;140:1474-1480.

21. Mari JJ, Williams P. A validity study of a psychiatric screening questionnaire (SRQ-20) in primary care in the city of São Paulo. Br J Psychiatry 1986;148:23-26.

22. Bertolucci PHF, Brucki SMD, Campacci SR, Juliano Y. O mini-exame do estado mental em uma população geral: impacto da escolaridade. Arq Neuropsiquiatr 1994;52:1-7.

23. World Health Organisation. ICD-10 classification of mental and behavioural disorders. Geneva: World Health Organisation, 1992.

24. Sherwin BB. Estrogen effects on cognition in menopausal women. Neurology 1997;48(Suppl 7):S21-S26.

25. Halbreich U. Role of estrogen in postmenopausal depression. Neurology 1997;48(Suppl 7):S16-S20.

26. Wickelgren I. Estrogen stakes claim to cognition. Science 1997;276:675-678.

27. Tang MX, Jacobs D, Stern Y, et al. Effect of oestrogen during menopause on risk and age at onset of Alzheimer's disease. Lancet 1996;348:429-432.

28. Almeida OP, Forlenza OV, Lima NKC, et al. Psychiatric morbidity among the elderly in a primary care setting: report from a survey in São Paulo, Brazil. Int J Geriat Psychiatry 1997;12:728-736. 
29. Hänninen T, Koivisto K, Reinikainen KJ, et al. Prevalence of ageing-associated cognitive decline in an elderly population. Age Ageing 1996;25:201-205.

30. Veras RP. País jovem com cabelos brancos: a saúde do idoso no Brasil. Rio de Janeiro: Relume Dumara, 1994.

31. Tobiansky R, Blizard R, Livingston G, Mann A. The Gospel Oak study stage IV: the clinical relevance of subjective memory impairment in older people. Psychol Med 1995;25:779-786.

32. Jorm AF, Christensen H, Henderson AS, et al. Complaints of cognitive decline in the elderly: a comparison of report by subjects and informants in a community survey. Psychol Med 1994;24:365-374.

33. O'Connor DW, Pollitt PA, Roth M, et al. Memory complaints and impairment in normal, depressed, and demented elderly persons identified in a community survey. Arch Gen Psychiatry 1990;47:224-227.

34. Crook TH. Diagnosis and treatment of memory loss in older patients who are not demented. In Levy R, Howard R, Burns A (eds). Treatment and care in old age psychiatry. Petersfield: Wrightson Biomedical Publ, 1993:95-111.

35. Green J, Goldstein FC, Sirockman BE, Green RC. Variable awareness of deficits in Alzheimer's disease. Neuropsychiat Neuropsychol Behav Neurol 1993;6:159-165.

36. Hänninen T, Hallikainen M, Koivisto K, et al. A follow-up study of age-associated memory impairment: neuropsychological predictors of dementia. JAGS 1995;43:1007-1015.

37. Helkala EL, Koivisto K, Hänninen T, et al. Stability of age-associated memory impairment during a longitudinal populationbased study. JAGS 1997;45:120-121.

38. O'Brien JT, Beats B, Hill K, et al. Do subjective memory complaints precede dementia? A three-year follow-up of patients with supposed 'benign senescent forgetfulness'. Int J Geriat Psychiatry 1992;7:481-486.

39. Spanó A, Forstl H, Almeida OP, Levy R. Neuroimaging and the differential diagnosis of early dementia: quantitative CT scan analysis in patients attending a memory clinic. Int J Geriat Psychiatry 1992;7:879-883.

40. Convit A, de Leon MJ, Tarshish C, et al. Hippocampal volume losses in minimally impaired elderly. Lancet 1995;345:266.

41. Parnetti L, Lowenthal DT, Presciutti O, et al. 1H-MRS, MRI-based hippocampal volumetry, and 99mTc-HMPAO-SPECT in normal aging, age-associated memory impairment, and probable Alzheimer's disease. JAGS 1996;44:133-138.

42. Reiman EM, Caselli RJ, Yun LS, et al. Preclinical evidence of Alzheimer's disease in person homozygous for the e4 allele for apolipoprotein E. N Engl J Med 1996; 334:752-758. 